\title{
Effects of Clarithromycin-Containing Quadruple Therapy on Helicobacter Pylori Eradication after Nitroimidazole-Containing Quadruple Therapy Failure
}

\author{
Hafez Fakheri ${ }^{1}$, Zeinab Bakhshi ${ }^{2}$, Zohreh Bari ${ }^{3^{*}}$, Saman Alhooei ${ }^{3}$
}

1. Professor, Department of Gastroenterology, Gut and Liver Research Center, Mazandaran University of Medical Sciences, Sari, Iran

2. Student of Medicine, Mazandaran University of Medical Sciences, Sari, Iran

3. Fellow of Gastroenterology, Mazandaran University of Medical Sciences, Sari, Iran

\footnotetext{
* Corresponding Author:

Zohreh Bari, MD

Gut and Liver Research Center, Mazandaran University of Medical Sciences, 48166 33131, Sari, Iran

Tel: + 981133350670

Fax: +98 1133363754

Email: zohreb252@yahoo.com

Received: 20 Jul. 2015

Accepted: 11 Oct. 2015
}

\section{ABSTRACT}

\section{BACKGROUND}

Several large clinical trials and meta-analyses have shown about $20 \%$ failure to eradicate Helicobacter pylori (H.pylori), necessitating investigations for second-line treatments. The aim of this study was to evaluate the effects of clarithromycin-containing quadruple regimen after nitroimidazole-containing quadruple therapy failure.

\section{METHODS}

Thirty two patients who had failed 10-day H.pylori treatment with omeprazole, amoxicillin, bismuth subcitrate, and metronidazole (OABM) regimen and 31 patients who had failed 10-day treatment with omeprazole, amoxicillin, bismuth subcitrate, and furazolidone (OAMF) regimen entered the study. They all received omeprazole $(20 \mathrm{mg})$, amoxicillin (1 gr), bismuth subcitrate $(240 \mathrm{mg})$ and clarithromycin $(500 \mathrm{mg})$ twice a day for 10 days. Eight weeks after treatment, H. pylori eradication was assessed by ${ }^{14} \mathrm{C}$-urea breath test.

\section{RESULTS}

Totally 61 patients completed the study. According to intention to treat (ITT) analysis, eradication rates by second-line OABC regimen were $84.37 \%(95 \%$ $\mathrm{CI}=71.7-96.9 \%)$ in OABM group and $77.41 \%(95 \% \mathrm{CI}=62.71-92.11 \%)$ in OABF group $(p=0.756)$. Per-protocol $(\mathrm{pp})$ eradication rates were $87.09 \%(95 \%$ $\mathrm{CI}=75.2-98.8 \%)$ and $82.75 \%(95 \% \mathrm{CI}=79.4-96 \%)$, respectively $(p=0.638)$. Also the cumulative eradication rates by $\mathrm{OABC}$ regimen were $80.9 \%(95 \% \mathrm{CI}=$ $71.2-90.6 \%)$ and $85 \%(95 \% \mathrm{CI}=75.9-94 \%)$ according to ITT and PP analyses, respectively. Severe side effects were reported in $3.1 \%$ of the patients.

\section{CONCLUSION}

Regarding ideal eradication rate $(>80 \%)$ and very low adverse effects, it seems that clarithromycin-containing quadruple therapy can be an encouraging regimen after nitroimidazole-containing regimen failure.

\section{KEYWORDS}

Helicobacter pylori; Clarithromycin; Metronidazole; Furazolidone

Please cite this paper as:

Fakheri H, Bakhshi Z, Bari Z. Effects of Clarithromycin-Containing Quadruple Therapy on Helicobacter Pylori Eradication after Nitroimidazole-Containing Quadruple Therapy Failure. Disease Middle East J Dig Dis 2015;8:51-6. DOI :10.15171/mejdd.2016.07

\section{INTRODUCTION}

Helicobacter pylori (H.pylori) infection is a global health problem and almost half of the population worldwide is infected by the organism. ${ }^{1}$ H.pylori infection is associated with peptic ulcer disease, gastric 
adenocarcinoma, and lymphoma. ${ }^{1}$

Although many investigators have tried to introduce regimens with ideal eradication rates, several large clinical trials and meta-analyses have shown about $20 \%$ failure to eradicate the infection, necessitating investigations for second-line treatments. ${ }^{2-5}$

According to Maastricht IV Consensus Report, in areas of low clarithromycin resistance $(<20 \%)$, clarithromycin-containing triple therapy or bismuth-containing quadruple regimen is recommended as the first line treatment. In case of failure, either a bismuth-containing quadruple regimen (if not used previously) or levofloxacin-containing triple therapy is recommended. On the other hand, in areas of high clarithromycin resistance, bismuthcontaining quadruple regimen is the first-line option and in case of unavailability, sequential therapy or any non-bismuth-containing quadruple regimen can be used. However, after failure, levofloxacincontaining triple therapy is recommended. ${ }^{6}$

As a general rule, second-line regimen should include at least one different antibiotic than what was previously prescribed. ${ }^{7}$ However, the global rise in antibiotic resistance for H.pylori eradication and unavailability of new effective antibiotics have led to use different combinations of the currently available antibiotics.

In Iran, resistance to metronidazole has been growing during the previous years and rates as high as $70 \%$ have been reported. ${ }^{8}$ Therefore, use of quadruple therapy, replacing metronidazole for furazolidone, is a frequent strategy. However, in case of failure, very few studies have evaluated the secondline options for H.pylori eradication. ${ }^{9-11}$ Therefore, we designed a study to evaluate the effects of clarithromycin-containing quadruple regimen on two groups of patients who had failed their previous treatment with a nitroimidazole- (metronidazole or furazolidone) containing quadruple therapy.

\section{MATERIALS AND METHODS}

Sixty three patients with positive test for H.pylori and peptic ulcer disease or gastric/duodenal erosions entered the study to receive a second-line H.pylori eradication regimen. Thirty two of the pa- tients had been previously treated with omeprazole $20 \mathrm{mg}$, amoxicillin $1 \mathrm{~g}$, bismuth subcitrate $240 \mathrm{mg}$, and metronidazole $500 \mathrm{mg}$ (OABM), all twice a day, for 10 days and the remaining 31 patients had previously received omeprazole $20 \mathrm{mg}$, amoxicillin $1 \mathrm{~g}$, bismuth subcitrate $240 \mathrm{mg}$, and furazolidone $200 \mathrm{mg}$ (OAMF) twice a day for 10 days. All the patients had failed their first-line H.pylori eradication regimen, confirmed by ${ }^{14} \mathrm{C}$-urea breath test (UBT) 8 weeks after treatment (using urea composition made by Helicap Institute of Isotopes, Budapest, Hungary and a cartridge made by Heliprobe breath card, Kibion Uppsala, Sweden).

The exclusion criteria were: age less than 18 years, significant underlying liver/cardiac/pulmonary or renal diseases, pregnancy, breast feeding, any kind of malignancy, previous gastric surgery, coagulopathy, and previous history of allergic reactions to any of the medications used in this protocol and recent proton pump inhibitor or antibiotic consumption.

Also, demographic information, history of previous upper gastrointestinal bleeding (GIB), smoking habits, and drug history including non-steroidal anti-inflammatory drugs (NSAIDs) consumption were recorded in questionnaires.

All the 63 patients received omeprazole $(20 \mathrm{mg})$, amoxicillin (1 gr), bismuth subcitrate $(240 \mathrm{mg})$, and clarithromycin $(500 \mathrm{mg})$ (OABC) twice a day for 10 days. The protocol was approved in the Ethics Committee of Mazandaran University of Medical Sciences and written informed consents were obtained from all the patients.

During the treatment course, the patients recorded side effects of medications each day and were advised to call the doctor in case of severe side effects. After the treatment course, they were visited and were asked about their compliance to treatment and side effects of the treatment. The severity of side effects were classified as: no side effect, mild (not interfering with daily activities), moderate (partially interfering with daily activities), and severe (abandoning daily activities). The compliance to treatment was considered as: excellent if the patient had used more than $90 \%$ of the prescribed drugs, good if the patient had used $80-90 \%$ of the 
medications, and poor in case of taking less than $80 \%$ of the prescribed drugs. Eight weeks after the second-line treatment, H.pylori eradication was reassessed using ${ }^{14} \mathrm{C}$-UBT.

Data were analyzed using SPSS software (version 16). To calculate the intention to treat eradication rate, all the patients who entered the study were considered and to calculate per-protocol eradication rate, only those who completed the entire protocol with more than $90 \%$ compliance to treatment were considered for analyses. Also $t$ test, fisher exact test, and univariate logistic regression analyses were used as appropriate. $p$ values less than 0.05 were considered as statistically significant.

\section{RESULTS}

In the initial study, 510 patients with H.pylori infection had entered the study. They were all naïve to H.pylori treatment. Two hundred and sixty patients had received the mentioned OABF regimen and 250 patients had received OABM. The per-protocol eradication rates were $87.4 \%$ and $82.7 \%$, respectively. Out of those patients who had failed treatment with either $\mathrm{OABF}$ or $\mathrm{OABM}$ therapies, 63 patients were enrolled in the present second-line treatment.

Thirty two patients were previously treated with OABM and 31 had previously received OABF. The mean ages of the patients were $45.6 \pm 8.7$ years and $45.8 \pm 12.5$ years in the two groups, respectively. Baseline demographic and clinical characteristics of the patients were not significantly different between the two groups (table 1). Sixty one patients completed the study. One patient from each group was lost to follow-up due to not performing UBT. Furthermore, one of the patients from OABF group discontinued treatment because of severe metallic taste and nausea.

Compliance to treatment was excellent in $100 \%$ of the patients in OABM group and $96.8 \%$ of the patients in OABF group. The compliance rates were not statistically different between the two groups.

According to intention to treat (ITT) analysis, eradication rates were $84.37 \%(27 / 32)(95 \% \mathrm{CI}=$ $71.7-96.9 \%)$ in the group that had previously received OABM, and $77.41 \%(24 / 31)(95 \% \mathrm{CI}=$
62.71-92.11\%) in OABF group ( $p=0.756)$. Per-protocol (pp) eradication rates were $87.09 \%(27 / 31)$ $(95 \% \mathrm{CI}=75.2-98.8 \%)$ and $82.75 \%(24 / 29)(95 \%$ $\mathrm{CI}=79.4-96 \%$ ), respectively $(p=0.638$ ) (figure 1 ). Also the cumulative eradication rates by OABC regimen were $80.9 \%(95 \% \mathrm{CI}=71.2-90.6 \%)$ and $85 \%(95 \% \mathrm{CI}=75.9-94 \%)$ according to ITT and PP analyses, respectively.

Totally, eight patients (12.6\%) reported side effects of the treatment, but they were mostly mild (table 2). Two patients (3.1\%) reported severe side effects, one of whom stopped treatment because of severe metallic taste and nausea.

\section{DISCUSSION}

An ideal second-line H.pylori eradication regimen is a regimen that can achieve more than $80 \%$ per-protocol eradication rate with less than $5 \%$ severe adverse effects. ${ }^{12}$ In our study, the clarithromycin-containing quadruple therapy could achieve more than $80 \%$ per-protocol eradication rates in both treatment arms and the cumulative eradication rate was $85 \%$. Also, the total rate of severe side effects was $3.1 \%$. Furthermore, $98.3 \%$ of the patients had excellent compliance to treatment.

Second-line H.pylorii eradication regimen must be chosen according to the patterns of regional antibiotic resistance and the type of first-line eradication regimen. It is recommended that if the first-line strategy was a nitroimidazole-containing quadruple regimen, a second-line clarithromycin-based regimen may be used afterwards, and vice versa. ${ }^{13-16}$ The absence of cross reaction between clarithromycin and nitroimidazole supports this concept.

Amongst very few reports evaluating second-line eradication regimens in Iran, the only study that has evaluated the effects of second-line clarithromycincontaining quadruple regimen was conducted by Minakari and colleagues in 2010. In the mentioned study, 220 patients who had failed treatment with $\mathrm{OABM}$ were randomized to receive either $\mathrm{OABC}$ or OBAzOf (az: Azithromycin, Of: ofloxacin). Perprotocol eradication rates were $74.7 \%$ and $86.7 \%$, respectively. ${ }^{9}$

There are other reports in favor of substituting 
Table 1: Baseline demographic and clinical characteristics of the patients in the two groups

\begin{tabular}{llccc}
\hline Variables & & $\begin{array}{c}\text { OABM group } \\
(\mathbf{N}=\mathbf{3 2})\end{array}$ & $\begin{array}{c}\text { OABF group } \\
(\mathbf{N}=\mathbf{3 1})\end{array}$ & $\boldsymbol{p}$-value \\
\hline Age (years) & $45.6 \pm 8.7$ & $45.8 \pm 12.5$ & 0.92 \\
\hline Sex (Male/Female) & $17 / 15$ & $19 / 12$ & 0.51 \\
\hline History of GI bleeding (\%) & $1(3.12)$ & 0 & 0.98 \\
\hline History of NSAIDs consumption (\%) & $1(3.12)$ & $1(3.22)$ & 0.99 \\
\hline History of smoking (\%) & $2(6.25)$ & $2(6.45)$ & 0.98 \\
\hline & Gastric Erythema & $2(6.2)$ & $1(3.2)$ & \\
& Duodenal ulcer & $14(43.7)$ & $25(80.6)$ & \\
& Gastric ulcer & $4(12.5)$ & $2(6.4)$ & \\
Endoscopic & Erosive duodenopathy & $2(6.2)$ & 0 & 0.061 \\
findings (\%) & Erosive gastropathy & $4(12.5)$ & $3(9.6)$ & \\
& Erosive gastro-duodenopathy & $1(3.1)$ & 0 & \\
& Familial history of gastric cancer & $4(12.5)$ & 0 & \\
& Gastric polyp & $1(3.1)$ & 0 & \\
\hline
\end{tabular}

GI: gastrointestinal, NSAID: Non-steroidal anti-inflammatory drug

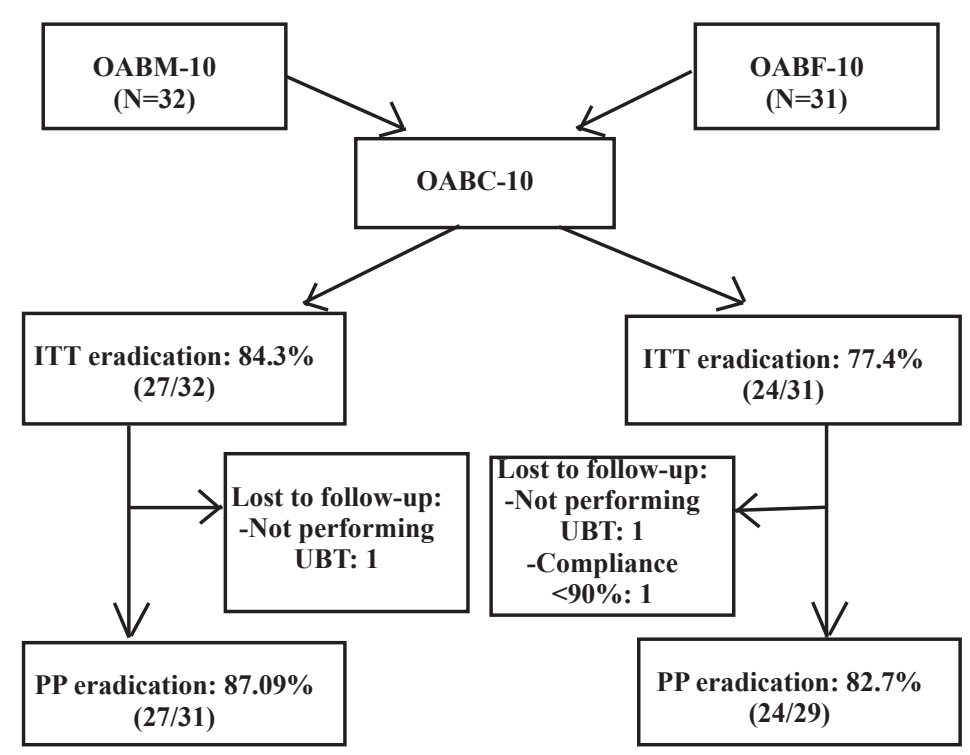

Fig. 1: Flow chart of treatment protocol and eradication rates

clarithromycin for metronidazole in case of failure to treat H.pylori. In this regard, Gisbert could achieve $85 \%$ eradication rate, ${ }^{17}$ Reilly and co-workers reported $86 \%,{ }^{18}$ and even Lerang and colleagues reported $100 \%$ H.pylori eradication rates by second-line clarithromycin-containing regimen after metronidazole failure. ${ }^{19}$ However, they all had used clarithromycincontaining triple therapy as the second-line strategy. But clarithromycin-containing triple therapy has shown disappointing results in our country, ${ }^{20-23}$ therefore, we used clarithromycin-containing "quadruple" regimen as the second-line treatment.

Furazolidone-containing quadruple regimens have also been evaluated as the first-line option in Iran and the reported results have mostly been ideal. ${ }^{24-28}$ However, in case of furazolidone failure, second-line studies are scanty. According to a review of different first-line or second-line H.pylori eradication regimens in Iran performed by Malekzadeh and co-workers, it is suggested that if a 
Table 2: Frequency of adverse effects and their severity during treatment

\begin{tabular}{|c|c|c|}
\hline Variables & & Frequency (\%) \\
\hline Diarrhea & & $1(1.5)$ \\
\hline Bloating & & $1(1.5)$ \\
\hline Metallic taste & & $3(4.7)$ \\
\hline Nausea & & $2(3.1)$ \\
\hline Vomiting & & $2(3.1)$ \\
\hline Headache & & $1(1.5)$ \\
\hline Malaise & & $1(1.5)$ \\
\hline Anorexia & & $1(1.5)$ \\
\hline Epigastric pain & & $1(1.5)$ \\
\hline Sleepiness & & $1(1.5)$ \\
\hline \multirow{3}{*}{$\begin{array}{l}\text { Total patients } \\
\text { with side effects }\end{array}$} & Mild & $5(7.9)$ \\
\hline & Moderate & $1(1.5)$ \\
\hline & Severe & $2(3.1)$ \\
\hline
\end{tabular}

furazolidone-containing regimen was used initially, a clarithromycin-based regimen can be used afterwards. ${ }^{29}$ Furthermore, since clarithromycin is an expensive drug in Iran, it seems better to keep this drug for second-line strategies.

The main limitation of our study was the unavailability of H.pylori culture. However, the resistance rates to clarithromycin have been less than $20 \%$ in most studies performed in Iran. ${ }^{30-35}$ Another limitation was the small sample size in each arm of the treatments. In fact, we had treated more than 700 patients with positive H.pylori test to achieve the present sample size.

In conclusion, since nitroimidazole-containing quadruple regimens are commonly used as firstline therapies in Iran, introducing a second-line regimen with ideal eradication rate and few adverse effects is worth considering. Accordingly, regarding ideal eradication rate $(>80 \%)$, excellent compliance to treatment and very low adverse effects, it seems that clarithromycin-containing quadruple therapy can be an encouraging regimen after nitroimidazole-containing regimen failure in Iran. We suggest further studies to evaluate the effects of other combinations of clarithromycin-containing regimens (such as concomitant or hybrid therapies) after nitroimidazole-containing regimen failure. Also, we suggest culture-guided strategies for sec- ond-line H.pylori treatment.

\section{CONFLICT OF INTEREST}

The authors declare no conflict of interest related to this work.

\section{REFERENCES}

1. Go MF. Review article: natural history and epidemiology of Helicobacter pylori infection. Aliment Pharmacol Ther 2002;16 Suppl 1:3-15. DOI:10.1046/j.13652036.2002.0160s1003.x.

2. Gisbert JP, González L, Calvet X, García N, López T, Roqué $\mathrm{M}$, et al. Proton pump inhibitor, clarithromycin and either amoxycillin or nitroimidazole: a metaanalysis of eradication of Helicobacter pylori. Aliment Pharmacol Ther 2000;14:1319-28. DOI:10.1046/j.13652036.2000.00844.x.

3. Gisbert JP, Pajares R, Pajares JM. Evolution of Helicobacter pylori therapy from a meta-analytical perspective. Helicobacter 2007;12 Suppl 2:50-8. DOI:10.1111/j.15235378.2007.00576.x.

4. Graham DY, Fischbach L. Helicobacter pylori treatment in the era of increasing antibiotic resistance. Gut 2010;59:1143-53. DOI:10.1136/gut.2009.192757.

5. Graham DY, Fischbach L. Sequential therapy for Helicobacter pylori infection: ethical considerations revisited. J Clin Gastroenterol 2010;44:523. DIO:10.1097/ MCG.0b013e3181e00566.

6. Malfertheiner P, Megraud F, O'Morain CA, Atherton J, Axon AT, Bazzoli F, et al. Management of Helicobacter pylori infection--the Maastricht IV/ Florence Consensus Report. Gut 2012;61:646-64. DOI:10.1136/ gutjnl-2012-302084.

7. Ferreira J, Moss SF. Current Paradigm and Future Directions for Treatment of Helicobacter pylori Infection. Curr Treat Options Gastroenterol 2014;12:373-84. DOI:10.1007/s11938-014-0027-6.

8. Talebi Bezmin Abadi A, Mobarez AM, Taghvaei T, Wolfram L. Antibiotic resistance of Helicobacter pylori in Mazandaran, North of Iran. Helicobacter 2010;15:505-9. DOI:10.1111/j.1523-5378.2010.00795.x.

9. Minakari M, Davarpanah Jazi AH, Shavakhi A, Moghareabed N, Fatahi F. A randomized controlled trial: efficacy and safety of azithromycin, ofloxacin, bismuth, and omeprazole compared with amoxicillin, clarithromycin, bismuth, and omeprazole as second-line therapy in patients with Helicobacter pylori infection. Helicobacter 2010;15:1549. DOI:10.1111/j.1523-5378.2009.00739.x.

10. Sotoudehmanesh R, Malekzadeh R, Vahedi H, Dariani NE, Asgari AA, Massarrat S. Second-line Helicobacter pylori eradication with a furazolidone-based regimen in patients who have failed a metronidazole-based regimen. Digestion 2001;64:222-5. DOI:10.1159/000048865.

11. Fakheri H, Bari Z, Sardarian H. A modified bismuth- 
containing quadruple therapy including a short course of furazolidone for Helicobacter pylori eradication after sequential therapy failure. Helicobacter 2012;17:264-8. DOI:10.1111/j.1523-5378.2012.00946.x.

12. Boyanova L, Mitov I. Geographic map and evolution of primary Helicobacter pylori resistance to antibacterial agents. Expert Rev Anti Infect Ther 2010;8:59-70. DOI:10.1586/ eri.09.113.

13. Gisbert JP. Rescue Therapy for Helicobacter pylori Infection 2012. Gastroenterol Res Pract 2012;2012:974594. DOI:10.1155/2012/974594.

14. Kobayashi I, Saika T, Muraoka H, Murakami K, Fujioka T. Helicobacter pylori isolated from patients who later failed H. pylori eradication triple therapy readily develop resistance to clarithromycin. J Med Microbiol 2006;55:737-40. DOI:10.1099/jmm.0.46316-0

15. Peitz U, Hackelsberger A, Malfertheiner P. A practical approach to patients with refractory Helicobacter pylori infection, or who are re-infected after standard therapy. Drugs 1999;57:905-20. DOI:10.2165/00003495-199957060-00006.

16. Deltenre M, Ntounda R, Jonas C, De Koster E. Eradication of Helicobacter pylori: why does it fail? Ital J Gastroenterol Hepatol 1998;30 Suppl 3:S326-8.

17. Gisbert JP, Boixeda D, Bermejo F, et al. Re-treatment after Helicobacter pylori eradication failure. Eur J Gastroenterol Hepatol 1999;11:1049-54.

18. Reilly TG, Ayres RC, Poxon V, Walt RP. Helicobacter pylori eradication in a clinical setting: success rates and the effect on the quality of life in peptic ulcer. Aliment Pharmacol Ther 1995;9:483-90. DOI:10.1111/j.1365-2036.1995.tb00410.x.

19. Lerang F, Moum B, Haug JB, et al. Highly effective second-line anti-Helicobacter pylori therapy in patients with previously failed metronidazole-based therapy. Scand J Gastroenterol 1997;32:1209-14. DOI: $10.3109 / 00365529709028149$.

20. Malekzadeh R, Merat S, Derakhshan MH, et al. Low Helicobacter pylori eradication rates with 4- and 7-day regimens in an Iranian population. $J$ Gastroenterol Hepatol 2003;18:13-7. DOI:10.1046/j.1440-1746.2003.02897.x.

21. Mirzaee V, Rezahosseini O. Randomized control trial: Comparison of Triple Therapy plus Probiotic Yogurt vs. Standard Triple Therapy on Helicobacter Pylori Eradication. Iran Red Crescent Med J 2012;14:657-66.

22. Sarkeshikian SS, Iranikhah A, Ghadir MR. Azithromycin based triple therapy versus standard clarithromycin based triple therapy in eradication of Helicobacter pylori infection in Iran: a randomized controlled clinical trial. The Turk J Gastroenterol 2013;24:10-4.

23. Taghavi SA, Jafari A, Eshraghian A. Efficacy of a new therapeutic regimen versus two routinely prescribed treatments for eradication of Helicobacter pylori: a randomized, double-blind study of doxycycline, co-amoxiclav, and omeprazole in Iranian patients. Digestive Dig Dis 2009;54:599-603. DOI:10.1007/s10620-008-0374-z.
24. Riahizadeh S, Malekzadeh R, Agah S, Zendehdel N, Sotoudehmanesh R, Ebrahimi-Dariani N, et al. Sequential metronidazole-furazolidone or clarithromycin-furazolidone compared to clarithromycin-based quadruple regimens for the eradication of Helicobacter pylori in peptic ulcer disease: a double-blind randomized controlled trial. Helicobacter 2010;15:497-504. DOI:10.1111/j.1523-5378.2010.00798.x.

25. Khatibian M, Ajvadi Y, Nasseri-Moghaddam S, et al. Furazolidone-based, metronidazole-based, or a combination regimen for eradication of Helicobacter pylori in peptic ulcer disease. Arch Iran Med 2007;10:161-7.

26. Fakheri H, Taghvaei T, Hosseini V, Bari Z. A comparison between sequential therapy and a modified bismuth-based quadruple therapy for Helicobacter pylori eradication in Iran: a randomized clinical trial. Helicobacter 2012;17:438. DOI:10.1111/j.1523-5378.2011.00896.x.

27. Fakheri H, Malekzadeh R, Merat S, Khatibian M, Fazel A, Alizadeh BZ, et al. Clarithromycin vs. furazolidone in quadruple therapy regimens for the treatment of Helicobacter pylori in a population with a high metronidazole resistance rate. Aliment Pharmacol Ther 2001;15:411-6. DOI:10.1046/j.1365-2036.2001.00931.x.

28. Fakheri H, Merat S, Hosseini V, Malekzadeh R. Lowdose furazolidone in triple and quadruple regimens for Helicobacter pylori eradication. Aliment Pharmacol Ther 2004;19:89-93. DOI:10.1046/j.1365-2036.2003.01822.x.

29. Malekzadeh. R, Mohamadnejad. M, Siavoshi F,. Treatment of helicobacter pylori infection in Iran. Arch Iran Med 2004;7:1-8

30. Safaralizadeh R, Siavoshi F, Malekzadeh R, Akbari MR, Derakhshan MH, Sohrabi MR, et al. Antimicrobial effectiveness of furazolidone against metronidazole-resistant strains of Helicobacter pylori. East Mediterr Health $J$ 2006;12:286-93.

31. Milani M, Ghotaslou R, Akhi MT, Nahaei MR, Hasani A, Somi MH, et al. The status of antimicrobial resistance of Helicobacter pylori in Eastern Azerbaijan, Iran: comparative study according to demographics. $J$ Infect Chemother 2012;18:848-52. DOI:10.1007/s10156-012-0425-4.

32. Mohammadi M, Doroud D, Mohajerani N, Massarrat S Helicobacter pylori antibiotic resistance in Iran. World $J$ Gastroenterol 2005;11:6009-13. DOI:10.3748/wjg.v11. i38.6009.

33. Shokrzadeh L, Jafari F, Dabiri H, Baghaei K, Zojaji H, Alizadeh AH, et al. Antibiotic susceptibility profile of Helicobacter pylori isolated from the dyspepsia patients in Tehran, Iran. Saudi J Gastroenterol 2011;17:261-4. DOI:10.4103/1319-3767.82581.

34. .Zendedel A, Moradimoghadam F, Almasi V, Zivarifar H. Antibiotic resistance of Helicobacter pylori in Mashhad, Iran. J Pak Med Assoc 2013;63:336-9.

35. Siavoshi F, Saniee P, Latifi-Navid S, Massarrat S, Sheykholeslami A. Increase in resistance rates of $\mathrm{H}$. pylori isolates to metronidazole and tetracycline--comparison of three 3-year studies. Arch Iran Med 2010;13:177-87. 